\title{
Fine Tuning of Track Impact Parameter Resolution of the DELPHI Detector ${ }^{\star}$
}

\author{
G.Borisov \\ Institute for High Energy Physics, Protvino, Russian Federation \\ C.Mariotti \\ INFN - section Sanità, Rome, Italy
}

\begin{abstract}
The fine tuning of the charged track impact parameter resolution for data and simulation in the DELPHI detector at LEP is described. This tuning was implemented in the software for the tagging of $B$ hadrons and has been applied in many precise measurements.
\end{abstract}

\footnotetext{
${ }^{\star}$ This work was partially supported by INTAS, contract INTAS-93-3602
} 



\section{Introduction}

The use of the precise DELPHI vertex detector $[1,2]$ greatly enhances the measurement of physical processes in the heavy quark sector, enabling accuracies of close to $1 \%$ to be achieved $([3,4,5])$. Almost all such measurements rely on a comparison of the observed data distributions with those predicted by a detailed detector simulation. For this comparison both the generation of the intrinsic physical processes and the simulation of detector response must be as realistic as possible.

The selection and the study of events containing $B$ hadrons are based on the separation of their origin and decay points, which produces an offset of the daughter particle tracks with respect to the point of the primary interaction. This is why for these studies the charged track impact parameter ${ }^{1}$ resolution is the most relevant and extremely crucial part of the detector response. The description of this resolution can significantly influence the physical result and the value of the systematic uncertainty.

The generated events in the DELPHI experiment are passed through the detector simulation package [6] and the same reconstruction program [7] as for the data. However, after this procedure some disagreement between data and simulation in the track resolution description remains. It is not drastically large but nevertheless can spoil precise measurements. This difference can be clearly seen, for example, in the distribution of the lifetime signed impact parameter $[8,3]$ shown in Fig.1. The impact parameter is signed positive (negative), if the track crosses the jet axis, to which it belongs, in front of (behind) the primary vertex. It should be noted that the difference between data and simulation is observed not only for tracks with negative impact parameters, where the distribution is dominated by the inaccuracy in the track reconstruction, but also for tracks with positive impact parameters, which contain the lifetime information of their parent and are used in the physical analysis.

The most important measured quantity used for the selection of events with $B$ hadrons is the track impact parameter significance, i.e. the ratio of the impact parameter to its error [8]. The disagreement between data and simulation in the distribution of this variable can be seen in Fig.2. Any disagreement here directly results in a large discrepancy in the tagging of $B$ hadrons. Fig.3a shows the ratio of data to simulation of the distribution of the special tagging variable $-\log _{10}\left(P_{E}^{+}\right)$, used in the DELPHI experiment for the selection of B-events (c.f. $[3,8]$ ). The variable $P_{E}^{+}$gives the probability that all tracks in the event with positive impact parameters originate from the primary vertex. For events with $B$ hadrons this probability is very low, while for light quark events it is randomly distributed between 0 and 1 . As can be seen from Fig.3a, for large values of $-\log _{10}\left(P_{E}^{+}\right)$, corresponding to purer samples of $B$ events, the difference between data and simulation becomes very significant.

In this note we describe a method for the correction of the detector resolution. The resulting improvement can be seen in Fig. 3b. The agreement between data and simulation is dramatically improved. The remaining differences can be at least partially explained by the uncertainties of modelling of the physical processes, which we did not attempt to correct. The improved agreement in the description of the tagging of $B$ hadrons is the main result of this work.

\footnotetext{
${ }^{1}$ The track impact parameter is defined as the distance of the track to the primary vertex at the point of its closest approach.
} 


\section{Parameterisation of the Track Resolution}

The tagging of $B$-hadrons can be done using a single detector measurement, namely the impact parameter of the track with respect to the primary vertex. Since the DELPHI vertex detector before 1994 was 2-dimensional [1], the computations are restricted to the plane perpendicular to the beam direction $(R-\phi$ plane). Therefore the detector resolution of the impact parameter in the $R-\phi$ plane is the only part of the detector response which needs to be corrected to improve the agreement between data and simulation. We do not consider below the angular or momentum resolution of the tracking system.

The resolution error of the reconstructed impact parameter in $R-\phi$ plane can be expressed in the following form:

$$
\begin{aligned}
\sigma^{2} & =a^{2}+b^{2} \cdot V(p, \vartheta) \\
V(p, \vartheta) & =\frac{1}{\left\{p \cdot \sin ^{\frac{3}{2}} \vartheta\right\}^{2}}
\end{aligned}
$$

In this equation $p$ is the momentum of the track in $\mathrm{GeV} / \mathrm{c}$ and $\vartheta$ is its polar angle.

The form of the parameterisation of the resolution error has direct physical motivation. The first term gives the intrinsic resolution of the tracking system in the absence of multiple scattering and is independent of the track parameters. The second term reflects the influence of multiple scattering in the material. It is inversely proportional to the momentum, which takes the form $p \sin \vartheta$ in the $R-\phi$ plane. It also has a proportional dependence on the square root of the distance traversed in the material. As most detectors in the barrel region are in the form of cylinders aligned along the beam direction, this distance is approximately proportional to $1 / \sin \vartheta$.

Fig. 4 shows the resolution error of the track impact parameter, which is given by the track fit, versus the function $V(p, \vartheta)$. The superimposed curve is defined by (1). As can be seen from this plot, the parameterisation (1) gives a reasonable description of the track resolution error.

The parameters $(a, b)$ depend in general on the pattern of the track measurements in the different parts of the tracking system. However in DELPHI this dependence can be considerably simplified since the track resolution is dominated by the vertex detector, which improves the resolution by one order of magnitude. Thus we can take into account the dependence of $(a, b)$ on the pattern of measurements in the vertex detector only. This statement is confirmed by Fig.4, which was constructed from all tracks with measurements in all three layers of the vertex detector; the variation of the resolution error inside this group of tracks is not big so that it can be parameterised by Eq.(1) with one set of parameters $(a, b)$. Since the vertex detector of DELPHI has three layers of sensitive planes[2], the number of different sets $(a, b)$ for tracks with at least 2 measurements in the vertex detector is equal to 4 and the problem of the description of the track resolution is reduced to the determination of parameters $(a, b)$ for 4 different groups of tracks.

\section{Determination of the Track Resolution}

It is obvious from Fig. 4 that the determination of the values $(a, b)$ for the parameterisation of the impact parameter resolution error (which will be denoted as $\sigma_{\text {res }}$ ) by Eq.(1) is simple 
and straightforward. However this impact parameter resolution error is merely the result of the fit of the track parameters. In this fit, model assumptions relating to the accuracy of the different parts of the tracking system and the material distribution in the detector are used. Thus the resolution error itself reflects not the real precision of the tracking system, but just our understanding of it. This understanding is unavoidably simplified to some extent and does not take into account all the effects which influence the reconstruction of the tracks.

The real accuracy of the tracking system is described by the observed distribution of the impact parameters. In the ideal case it should be gaussian with variance $\sigma_{o b s}$, which is parameterised by Eq.(1) as well. However a problem arises in the real data since the "true" impact parameter of the track with respect to its origin cannot be determined because the point of origin itself is not known. Most of the tracks come from the primary interaction, but there are some tracks from secondary interactions in the material or from decays of long-lived particles such as $B$-hadrons, $K_{S}^{0}$, hyperons etc. The fraction of such tracks can be significantly reduced by:

- rejecting particles from reconstructed $V^{0}$ decays;

- selecting events which pass an "anti- $B$ " cut on the $B$-tagging variable [8], e.g.: $-\log _{10}\left(P_{E}^{+}\right)>0.1$

- using tracks with negative impact parameters;

- selecting tracks with small absolute value of significance.

The remaining influence of the secondary tracks on the measurement of $\sigma_{o b s}$ can be checked in the simulation, where both impact parameters - the observed one (i.e. with respect to the primary vertex) and the "true" one (i.e. with respect to the origin of each given particle) - are known. The distribution of the "true" impact parameter reflects the effective accuracy of the tracking system and should be considered as the reference for all other resolution measurements. Table 1 gives the comparison of the values of $(a, b)$ for the parameterisation of the "true" and observed impact parameter distributions in simulation for tracks with measurements in all three layers of the vertex detector. The obtained values of $(a, b)$ are compatible within the statistical errors, which means that the procedure for their measurement is reliable and is not influenced by the secondary tracks. It is also seen that the parameterisation of the resolution error, coming from the track fit and shown in the third column of table 1, differs from that of the "true" impact parameters.

The parameterisation of the distribution of observed impact parameters is determined by a maximum likelihood fit. For each track the probability density function is defined by:

$$
\begin{aligned}
f(d) & =\frac{1}{\sqrt{2 \pi} \sigma_{d}} \exp \left\{-d^{2} /\left(2 \cdot \sigma_{d}^{2}\right)\right\} \\
\sigma_{d}^{2} & =\sigma_{o b s}^{2}(a, b)+\sigma_{p v}^{2}
\end{aligned}
$$

where $d$ is the track impact parameter, $\sigma_{o b s}$ is the function of $(a, b)$ as defined by Eq.(1) and $\sigma_{p v}$ is the error corresponding to the uncertainty in the primary vertex position. Note 
that if a given track is used for the determination of the primary interaction point, there will be a correlation between its impact parameter and the vertex position [8]. To remove this correlation, the impact parameter of such a track is measured relative to the primary vertex, which is determined without the use of that track.

The quality of the description of the impact parameter distribution can be checked from the distribution of track probability, defined by:

$$
P(d)=\int_{x>d} f(x) d x
$$

with the probability density function $f(x)$ given by Eq.(2). The distribution of $P(d)$ should be flat provided the selected parameterisation corresponds to the distribution of the impact parameter. Fig.5 shows the distributions of $P(d)$ for the tracks within three different momentum ranges. The $\chi^{2}$ of the fit of these distributions by a constant for $P(d)>0.08$ is also shown. The peak at zero probability reflects the non-gaussian tail of the impact parameter distribution, which is produced by the tracks of particles from secondary interactions in the material or from the decays of long-lived particles. Such tracks were removed from the maximum likelihood fit by requiring the track impact parameter $d$ to be less than $1.8 \cdot \sigma_{d}$ which corresponds to the condition $P(d)>0.08$. It seems that with such a selection, the probability distribution is flat enough and it can be seen that the parameterisation (1-2) is suitable for the description of the observed distribution of the impact parameter.

One more check of the parameterisation form (1-2) is shown in Fig.6, where the values of $(a, b)$ are given as a function of the track polar angle. These plots prove that there is not any significant remaining dependence of the coefficients $(a, b)$ on the polar angle within the acceptance of the DELPHI vertex detector.

In the ideal case, the resolution of the tracking system should be independent of the azimuthal angle $(\phi)$ of the track direction. This is found to be the case in the simulation. However the real data show some dependence of the resolution on $\phi$ which is displayed in Fig.7a. In this figure the average value of the track probability, defined similarly to Eq.(3), is shown as a function of the azimuthal angle $\phi$. The observed significant variation of the track resolution can be explained, for example, by inaccuracy in the azimuthal alignment of the tracking system. It can be taken into account by dividing the full range of $\phi$ into zones of approximately the same resolution, and performing the fit of the observed impact parameter distribution in each zone separately. It means that effectively the parameters $(a, b)$ become functions of $\phi$. As shown in Fig.7b, such a procedure corrects properly for the variation of track resolution with azimuthal angle.

\section{Tuning of Track Resolution}

As was shown in the previous section, two different estimates of the track resolution can be obtained: using the resolution error given by the track fit $\left(\sigma_{\text {res }}\right)$ or using the observed distribution of the track impact parameters $\left(\sigma_{o b s}\right)$. Both estimates can be parameterised by the same function (1) with slightly different coefficients. This difference comes mainly from the uncertainty in the prior knowledge of the detector precision, which propagates to the uncertainty in the resolution error $\sigma_{\text {res }}$. The value of $\sigma_{o b s}$ is an a posteriori estimate of resolution and due to this it is more accurate and sensitive to some effects such as the $\phi$ dependence of the resolution. On the other hand, the estimate of $\sigma_{o b s}$ is deduced from an 
average over some sample of tracks so that individual differences in the track resolution (e.g. due to the variation of the radius values of the track measurements) which are taken into account by $\sigma_{\text {res }}$ disappear in $\sigma_{o b s}$.

The correction of the track resolution is performed in such a way that it combines the better average description of the resolution by $\sigma_{o b s}$ with the individual peculiarities of the track reconstruction which are kept in $\sigma_{\text {res }}$. The resolution error of each track in data is multiplied by the factor $K_{\text {res }}^{R D}$ :

$$
\begin{aligned}
\sigma_{r e s}^{R D} & \rightarrow \sigma_{r e s}^{R D} \cdot K_{r e s}^{R D} \\
\left(K_{r e s}^{R D}\right)^{2} & =\frac{\left(a_{o b s}^{R D}\right)^{2}+\left(b_{o b s}^{R D}\right)^{2} \cdot V(p, \vartheta)}{\left(a_{r e s}^{R D}\right)^{2}+\left(b_{r e s}^{R D}\right)^{2} \cdot V(p, \vartheta)}
\end{aligned}
$$

In this equation, the function $V(p, \vartheta)$ is defined by Eq. $(1),\left(a_{o b s}^{R D}, b_{o b s}^{R D}\right)$ are the coefficients of parameterisation of $\sigma_{o b s},\left(a_{r e s}^{R D}, b_{r e s}^{R D}\right)$ are the coefficients of parameterisation of $\sigma_{\text {res }}$ and the label $(R D)$ denotes the data. After this modification the new resolution error is parameterised in a similar way to $\sigma_{o b s}$ with coefficients $\left(a_{o b s}^{R D}, b_{o b s}^{R D}\right)$.

The main goal of this study is the correction of the track resolution in the simulation. The track impact parameter error in the simulation can be easily corrected similarly to Eq.(4) multiplying it by the factor $K_{\text {res }}^{M C}$ :

$$
\begin{aligned}
\sigma_{r e s}^{M C} & \rightarrow \sigma_{r e s}^{M C} \cdot K_{r e s}^{M C} \\
\left(K_{r e s}^{M C}\right)^{2} & =\frac{\left(a_{o b s}^{R D}\right)^{2}+\left(b_{o b s}^{R D}\right)^{2} \cdot V(p, \vartheta)}{\left(a_{r e s}^{M C}\right)^{2}+\left(b_{r e s}^{M C}\right)^{2} \cdot V(p, \vartheta)}
\end{aligned}
$$

Here $\left(a_{r e s}^{M C}, b_{r e s}^{M C}\right)$ are the coefficients of the parameterisation of $\sigma_{\text {res }}$ in simulation.

However the correction of impact parameter error in the simulation is not sufficient because the distribution of the impact parameters itself differs from the data, as can be seen in Fig.1. Thus the track impact parameters in the simulation should be additionally smeared. It can be done as follows.

In the previous section it was noted that the distribution of "true" impact parameters (with respect to the point of origin for that particle) can be parameterised by a gaussian with the variance expressed in the form of Eq.(1) with coefficients $\left(a_{o b s}^{M C}, b_{o b s}^{M C}\right)$. The multiplication of the "true" impact parameter by the value of $K_{o b s}^{M C}$ :

$$
\left(K_{o b s}^{M C}\right)^{2}=\frac{\left(a_{o b s}^{R D}\right)^{2}+\left(b_{o b s}^{R D}\right)^{2} \cdot V(p, \vartheta)}{\left(a_{o b s}^{M C}\right)^{2}+\left(b_{o b s}^{M C}\right)^{2} \cdot V(p, \vartheta)}
$$

effectively transforms the variance $\sigma_{o b s}^{M C}$ of its distribution as: $\left(\sigma_{o b s}^{M C} \rightarrow \sigma_{o b s}^{R D}\right)$. This multiplication is equivalent to the addition of the value $\delta$ to the track impact parameter:

$$
\delta=d_{\text {true }} \cdot\left(K_{o b s}^{M C}-1\right)
$$

where $d_{\text {true }}$ is the "true" impact parameter.

After this transformation the variance of the impact parameter distribution is forced to be the same as in data. In addition, such a method of modification of the track impact parameter has the following important features: 
- The smearing of the track impact parameter in simulation is done without additional randomization;

- The correction is equivalently applied to both primary and secondary tracks;

- Because the values $\left(a_{o b s}^{R D}, b_{o b s}^{R D}\right)$ in fact are functions of the track azimuthal angle $\phi$ (see section 3 ), after this correction the resolution in simulation acquires the same $\phi$-dependence as in data.

The agreement between data and simulation becomes much better after applying the corrections (4-7) to the track impact parameter and to the corresponding error. Fig. 8 shows the ratio of the significance distributions of tracks with negative impact parameters. It should be compared with the same distribution without correction shown in Fig. $2 \mathrm{~b}$.

The corrections described above assume that the impact parameter distribution can be parameterised by a gaussian with variance $\sigma_{o b s}$. As can be seen from Fig.2, this is true only for small values of the significance. Indeed, in this part of the significance distribution, the agreement between data and simulation becomes especially good after applying the correction. The description of the non-gaussian tail is worse, which implies that some additional correction should be performed for this part of the distribution.

Unfortunately a complete, physically motivated parameterisation of the non-gaussian tail of the significance distribution does not exist since there are many sources of completely different nature which produce it. They include unavoidable mistakes in the track search algorithm producing large impact parameters, interactions of the particles with the detector material, decays of long-lived particles $\left(K^{0}, \Lambda\right)$, etc. It seems impossible to estimate in the data either the shape of the significance distribution from these sources or their relative fractions. That is why the parameterisation of the non-gaussian tail is rather arbitrary. We select the form which gives the best description of our data, although some different parameterisations are equally possible and were used in other experiments [9]. The parameterisation of the significance distribution is given by the function:

$$
\begin{aligned}
f(d) & =\frac{P_{1}}{\sqrt{2 \pi} \sigma_{o b s}} \exp \left\{\frac{-d^{2}}{2 \sigma_{o b s}^{2}}\right\}+\frac{P_{2}}{\sqrt{2 \pi} K_{s g} \sigma_{o b s}} \exp \left\{\frac{-d^{2}}{2\left(K_{s g} \sigma_{o b s}\right)^{2}}\right\}+ \\
& +\frac{P_{3} K_{e x p}}{2 \sigma_{o b s}} \exp \left\{-K_{e x p} \frac{|d|}{\sigma_{o b s}}\right\}
\end{aligned}
$$

In this equation $\left(P_{1}+P_{2}+P_{3}\right)=1$, the value of $\sigma_{o b s}$ is determined as described in section 3 and the last two terms parameterise the non-gaussian tail of the significance distribution.

According to Eq.(8) the impact parameter of tracks in simulation is modified in the following way. First of all the "gaussian" correction defined by Eq.(6-7) is applied to all the tracks. For a fraction $P_{2}$ of the tracks, the factor $K_{o b s}^{M C}$ is multiplied by $K_{s g}$ : ( $K_{o b s}^{M C} \rightarrow K_{o b s}^{M C} \cdot K_{s g}, s g$ stands for "second gaussian"). This modification takes into account the second term of Eq.(8). Similarly a fraction $P_{3}$ of the tracks is exponentially smeared around their generation point with a slope of exponent $K_{\text {exp }}$. The parameters $P_{2}, P_{3}, K_{s g}$ and $K_{e x p}$ are selected iteratively by comparing the modified significance distribution in the simulation with that in data. Because the correction of the gaussian part by Eq.(6-7) removes the largest part of the difference between data and simulation 
(see Fig.8), the fractions of tracks $P_{2}$ and $P_{3}$ subjected to non-gaussian modification numerically are very small and do not exceed a few percent. This is very important for physical applications as it reduces the uncertainty of the analyses due to the difference in resolution.

The comparison of the significance distribution after correction of the non-gaussian tail is given in Fig.9 which confirms that, after applying this procedure, the data and simulation agree in a wide range of significance values.

\section{$5 \quad$ Results and Conclusions}

After applying the tuning procedure described in this paper, the agreement between data and simulation is improved for all variables used in the identification of $B$ hadrons.

Fig.9 shows the comparison of track significance while Fig.10 shows the distribution of the signed impact parameter after tuning procedure. This figure should be compared with the similar distribution without tuning shown in Fig.1. The improvement of the description of the distribution of the $B$-tagging variable, shown in Fig.3, is also remarkable.

The tuning procedure also improves the agreement between data and simulation when applied in the secondary vertices search [10]. Fig.11 shows the distribution of distance from the beam-spot to the reconstructed secondary vertex divided by its error after the tuning of the track resolution. As can be seen from this figure, there is a good agreement not only for tracks from the primary interaction (negative length of secondary vertex), but for tracks from the secondary decays as well (positive length).

This tuning procedure has been applied in some precise measurements with the DELPHI detector [3, 4], where a significant reduction of the systematics due to inconsistency of data and simulation was achieved.

\section{Acknowledgements}

We would like to thank many our colleagues from the DELPHI collaboration, who tested the tuning procedure and helped to improve it. The contribution of A.Normand, who applied the tuning in the secondary vertex search, is especially important. We would like to thank J-E.Augustin, L.Lyons and M.Mazzucato for the support of this work and many useful discussions and P.Collins for editing of this paper. 


\section{References}

[1] N. Bingefors et al., Nucl. Inst. Meth. 328A, 447 (1993).

[2] V. Chabaud et al. The DELPHI Silicon Microvertex Detector with Double Sided Readout, preprint CERN-PPE/95-86, to be published in Nucl. Instr. and Meth.

[3] DELPHI Collaboration, Z.Phys. C 66, 323 (1995)

[4] DELPHI Collaboration, Z.Phys. C 65, 569 (1995)

[5] DELPHI Collaboration, Lifetimes of Charged and Neutral B Hadrons using Event Topology, preprint CERN-PPE/95-59 (1995)

[6] DELSIM reference Manual, DELPHI 87-98 PROG 100, Geneva, 1989

[7] DELANA User's Guide, DELPHI 89-44 PROG 137, Geneva, 1989

[8] G.Borisov, Lifetime Tag of Events with $B$-hadrons with the DELPHI Detector, preprint IHEP (Protvino) 94-98 (1994) and DELPHI 94-125 PROG 208, Geneva, 1994

[9] ALEPH Collaboration, Phys. Lett. B313, 535 (1993)

[10] A. Normand, private communication. 


\begin{tabular}{|c|c|c|c|}
\hline Parameter & "true" IP & observed IP & resolution error \\
\hline$a[\mu \mathrm{m}]$ & $21.7 \pm 0.2$ & $21.1 \pm 0.3$ & 18.4 \\
$b[\mu \mathrm{m}]$ & $59.1 \pm 0.4$ & $59.4 \pm 0.4$ & 61.5 \\
\hline
\end{tabular}

Table 1: Values of $(a, b)$ obtained from the fit of the "true" and the observed impact parameters in the simulation for the tracks with measurements in all three layers of the vertex detector. The third column gives the values $(a, b)$ of parameterisation of the resolution error coming from the track fit. 


\section{Figure Caption}

Fig.1: (a) The lifetime signed impact parameter distribution. The points with errors are real data, the histogram is simulation. (b) The ratio of these distributions (data divided by simulation).

Fig.2: (a) The significance distribution for tracks with negative impact parameters. The points with errors are real data, the histogram is simulation. (b) The ratio of these distributions (data divided by simulation).

Fig.3: The data to simulation ratio of the distribution of the logarithm of the positive event probability $\left(P_{E}^{+}\right)$(a) before the tuning of the detector resolution and (b) after the tuning.

Fig.4: The resolution error of the track impact parameter versus $p^{2} \sin ^{3}(\vartheta)$ for tracks with measurements in all three layers of the vertex detector.

Fig.5: The distribution of probability as defined by Eq.(3) for tracks with different momenta. The $\chi^{2}$ values are for the fit by a constant for probability greater than 0.08 .

Fig.6: The dependence of coefficients $(a, b)$ on the track polar angle.

Fig.7: (a) The dependence of the average track probability on the track azimuthal angle in the real data; (b) the same distribution after using $\phi$-dependent resolution.

Fig.8: The ratio of the significance distributions (data divided by simulation) for tracks with negative impact parameters after correction of the gaussian part of the resolution.

Fig.9: (a) The significance distribution for tracks with negative impact parameters after the correction of the resolution by Eq.(8). The points with errors are real data, the histogram is simulation. (b) The ratio of these distributions (data divided by simulation).

Fig.10: (a) The lifetime signed impact parameter distribution after correction of track resolution. The points with errors are real data, the histogram is simulation. (b) The ratio of these distributions (data divided by simulation).

Fig.11: (a) The distribution of the decay length divided by its error for secondary vertices after correction of track resolution. The points with errors are real data, the histogram is simulation. (b) The ratio of these distributions (data divided by simulation). 

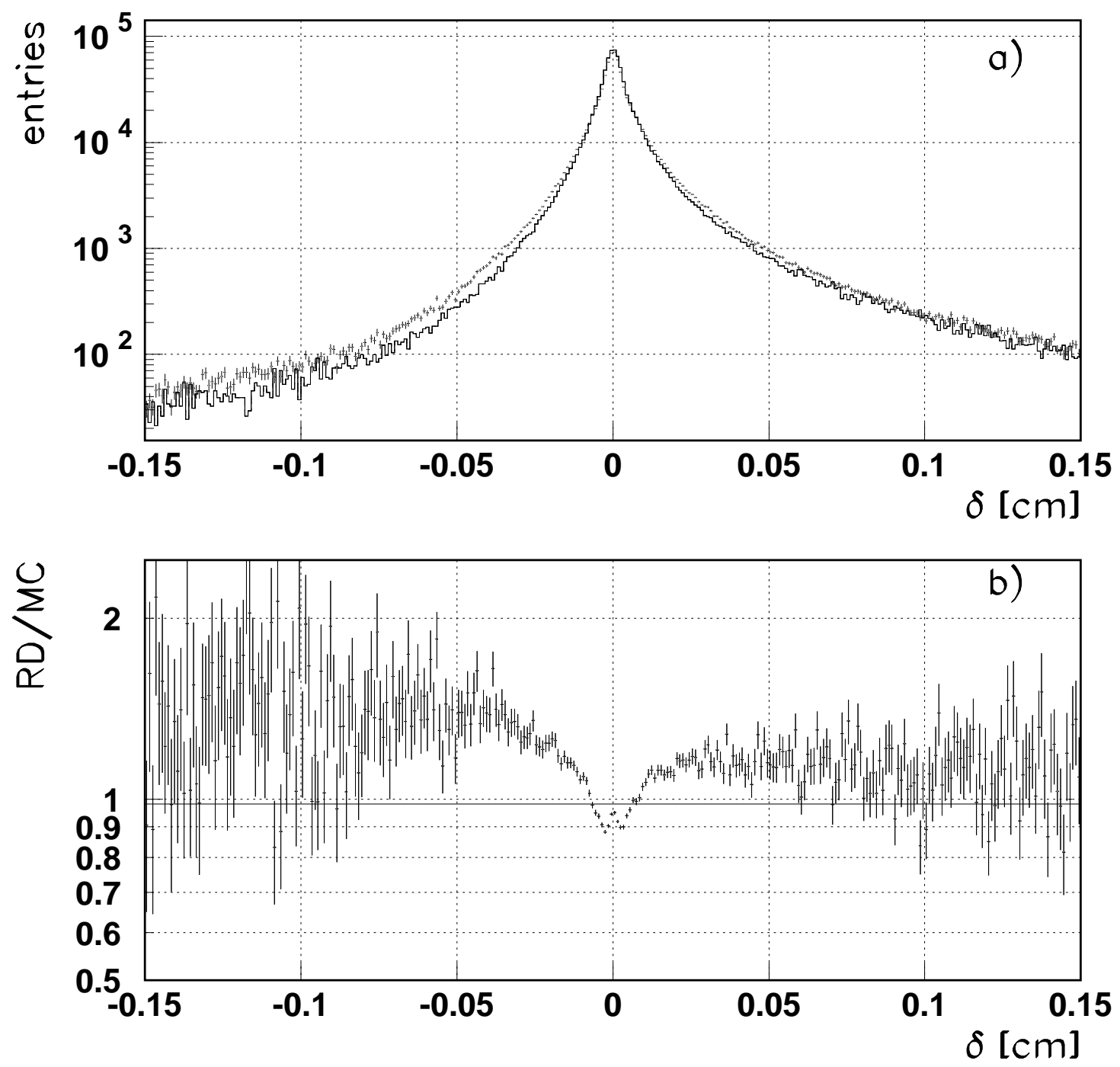

Figure 1: 

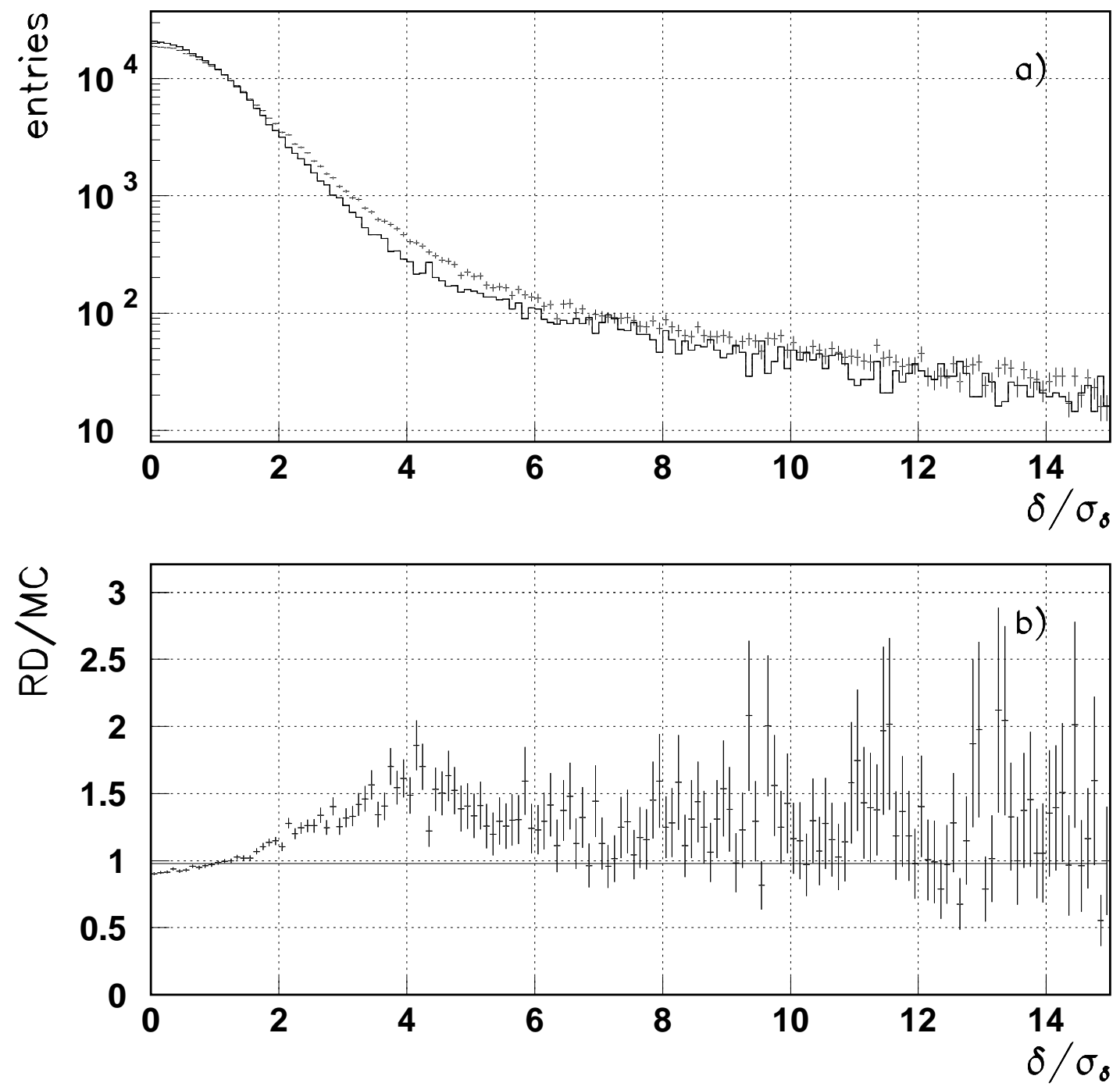

Figure 2: 

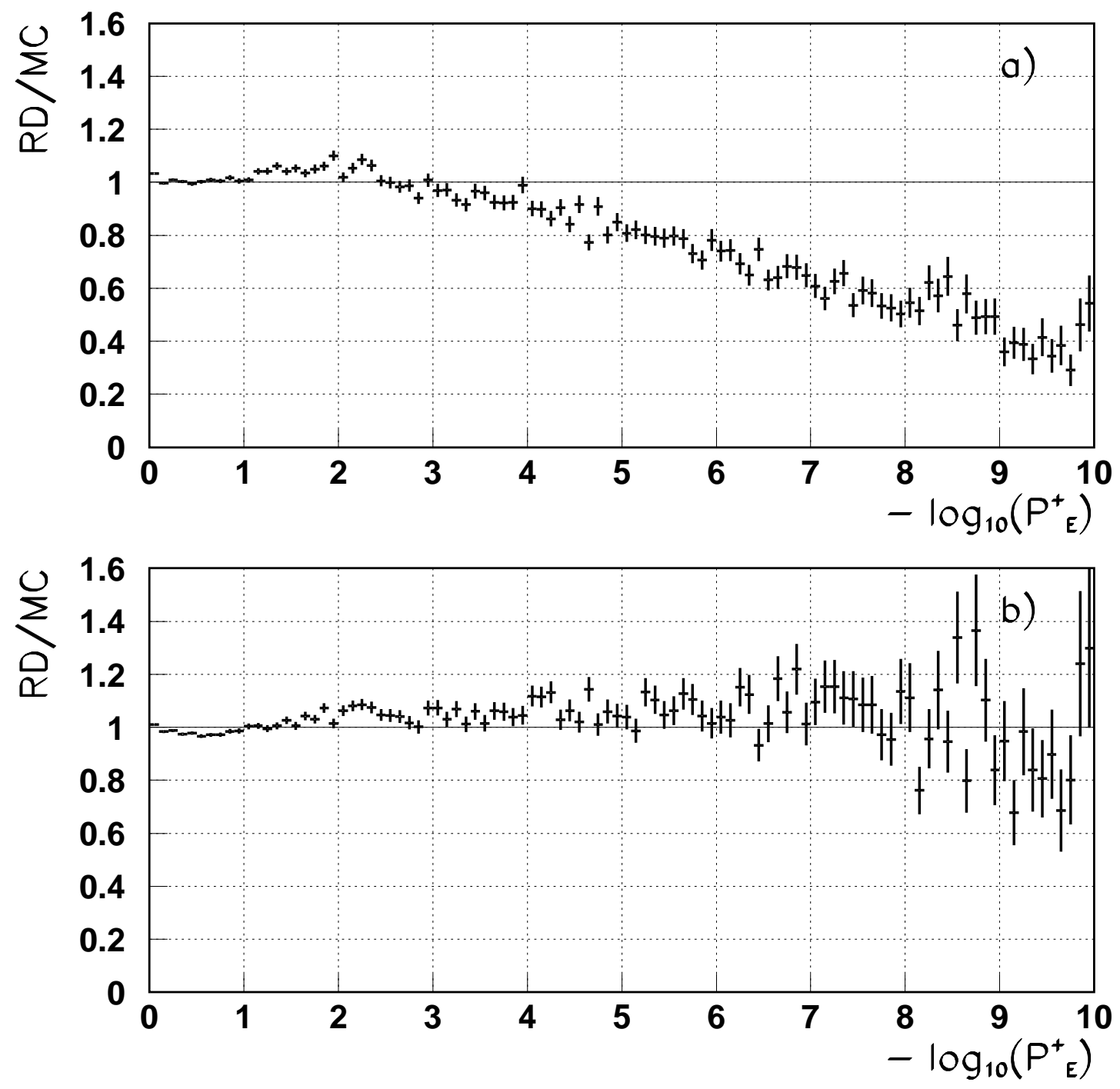

Figure 3: 


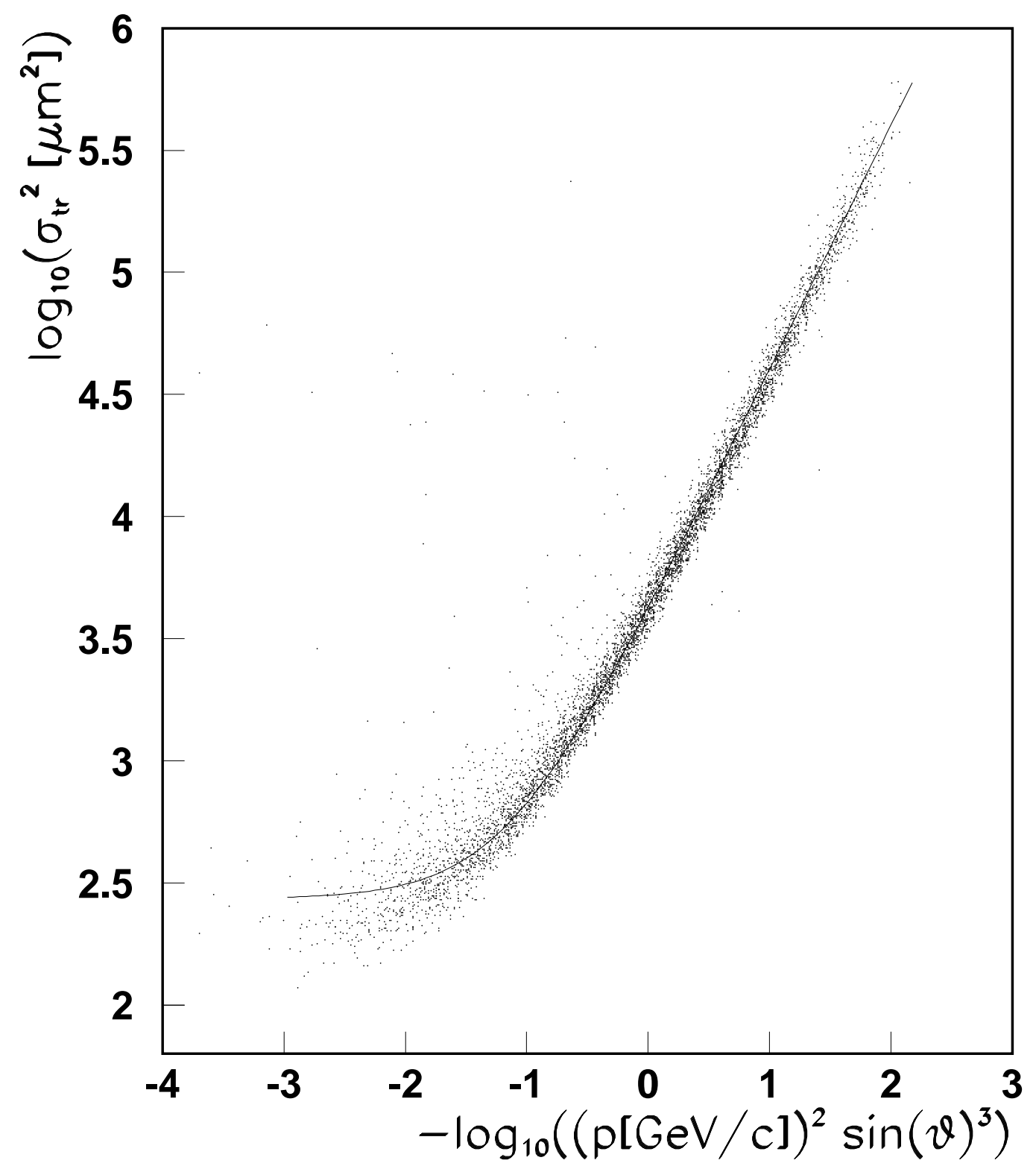

Figure 4: 


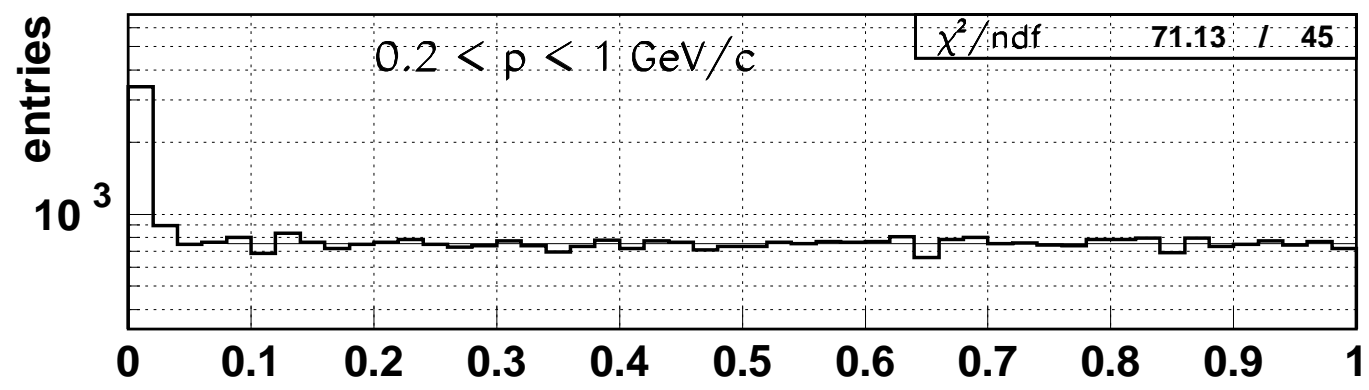

Probability
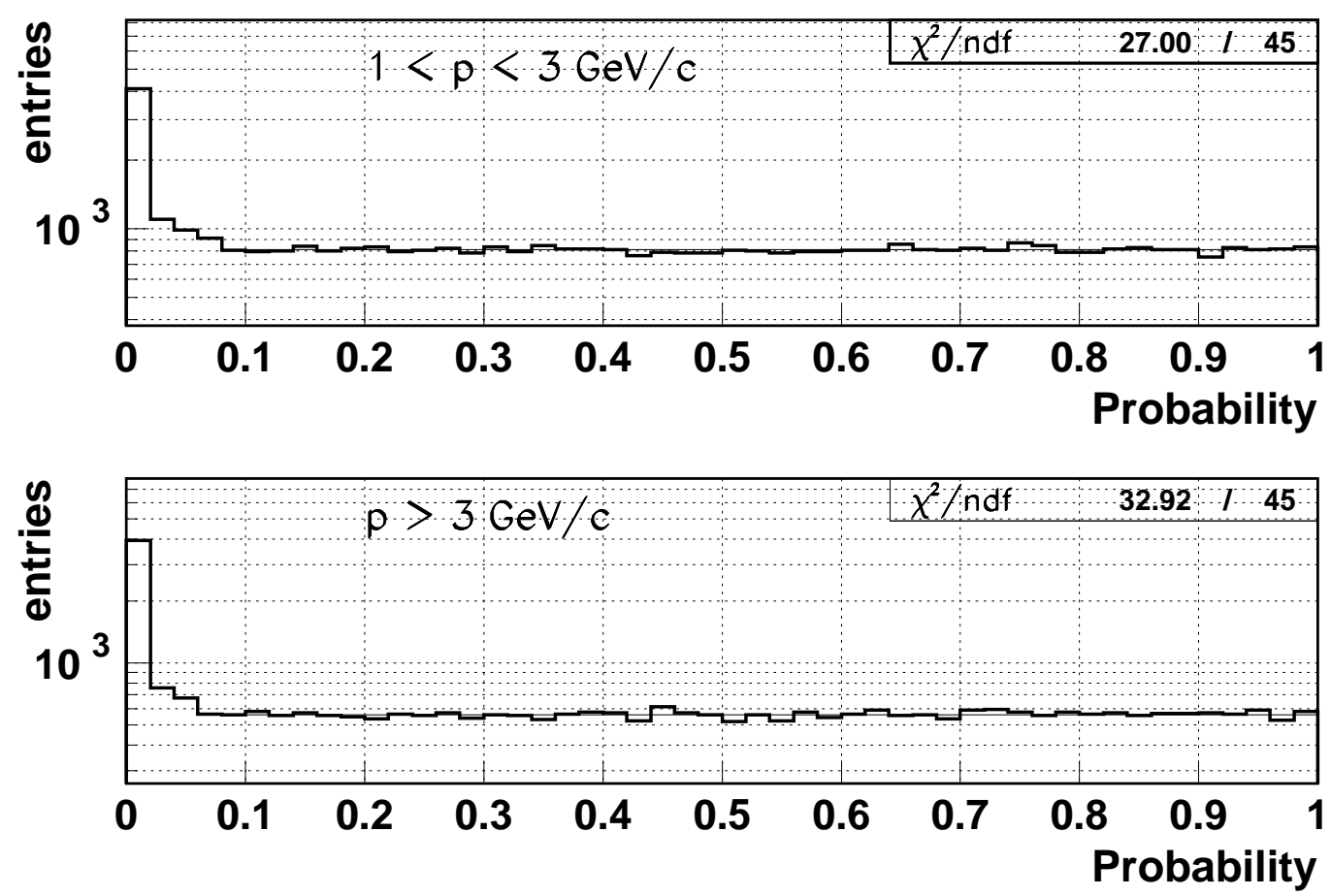

Figure 5: 

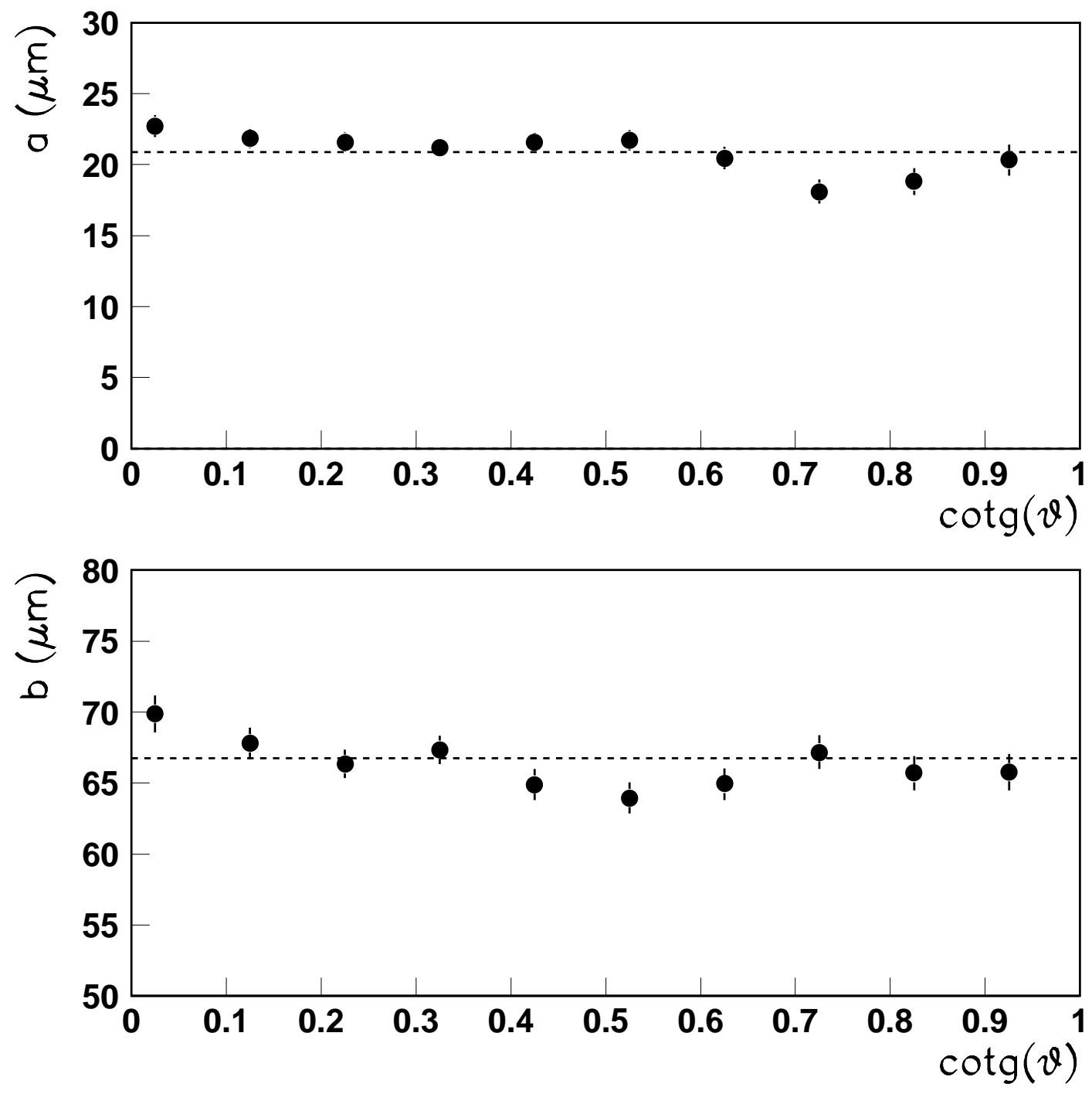

Figure 6: 

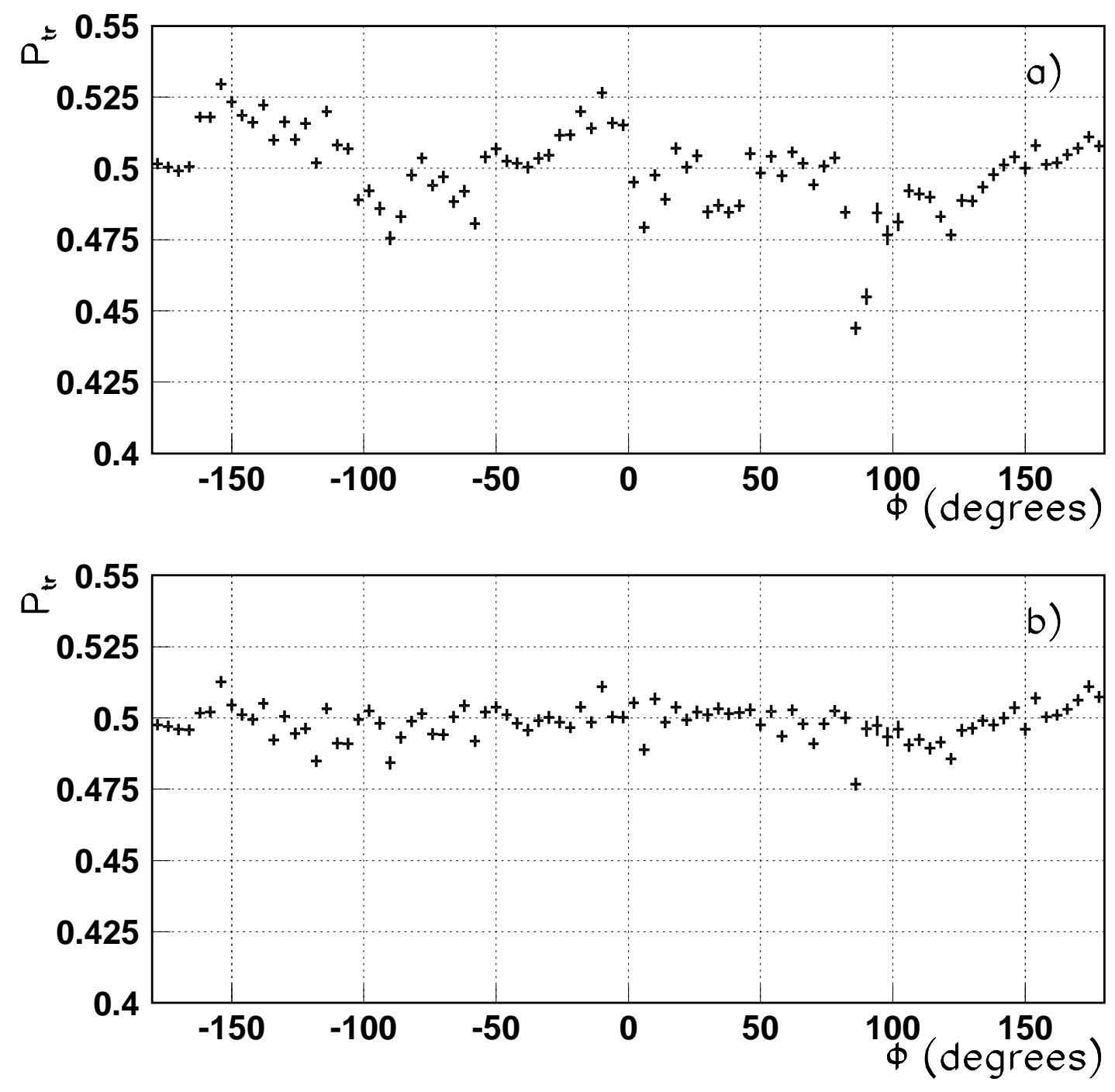

Figure 7: 


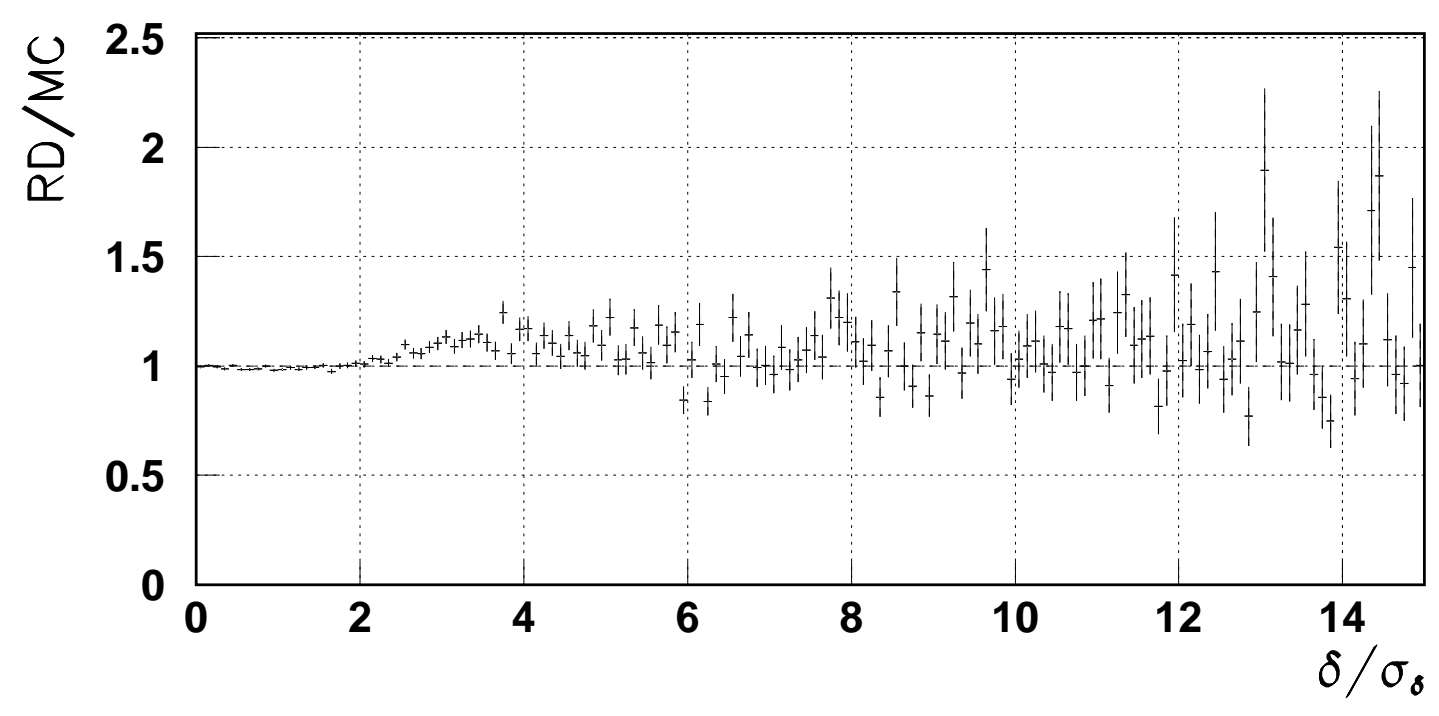

Figure 8: 

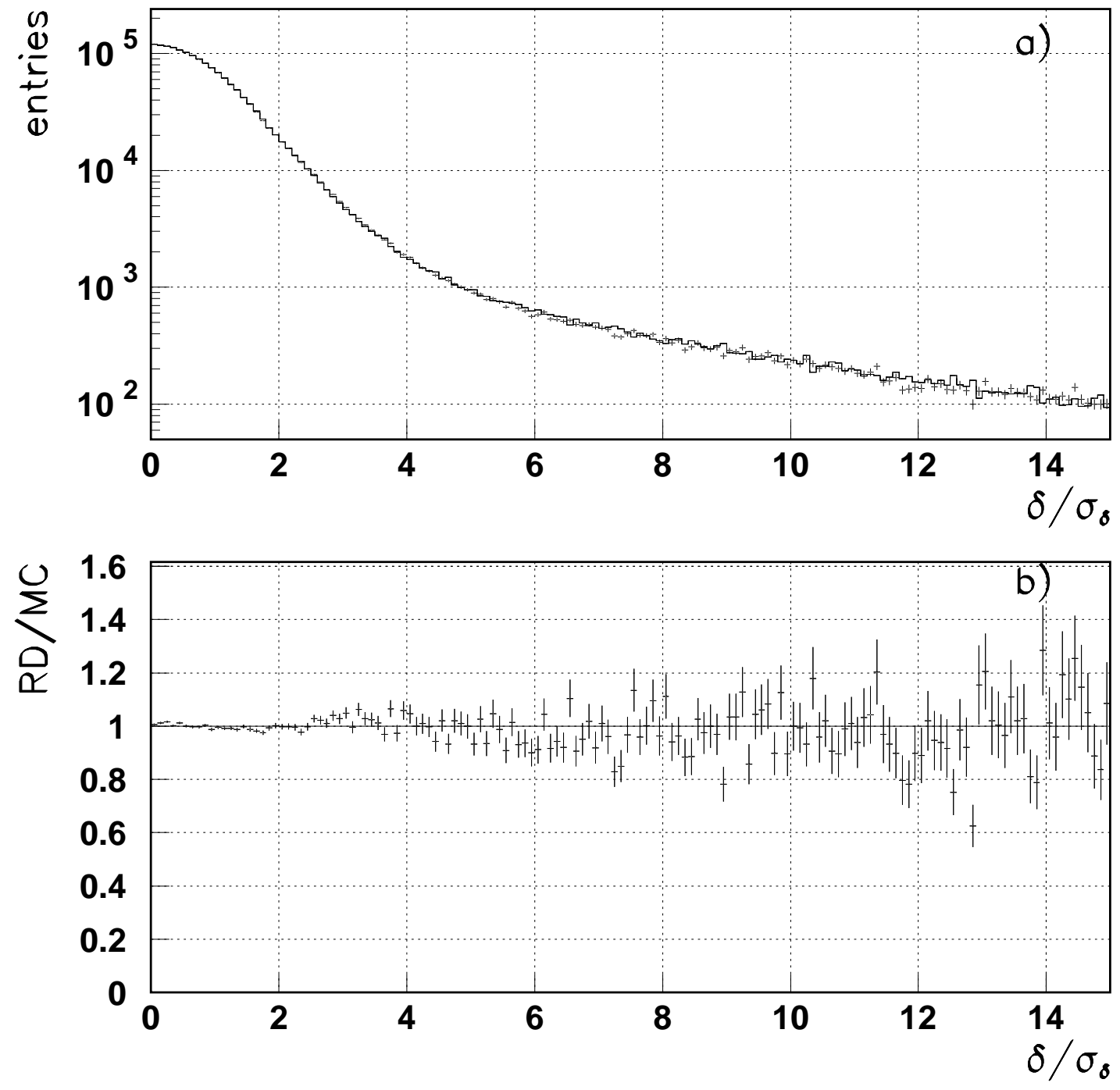

Figure 9: 

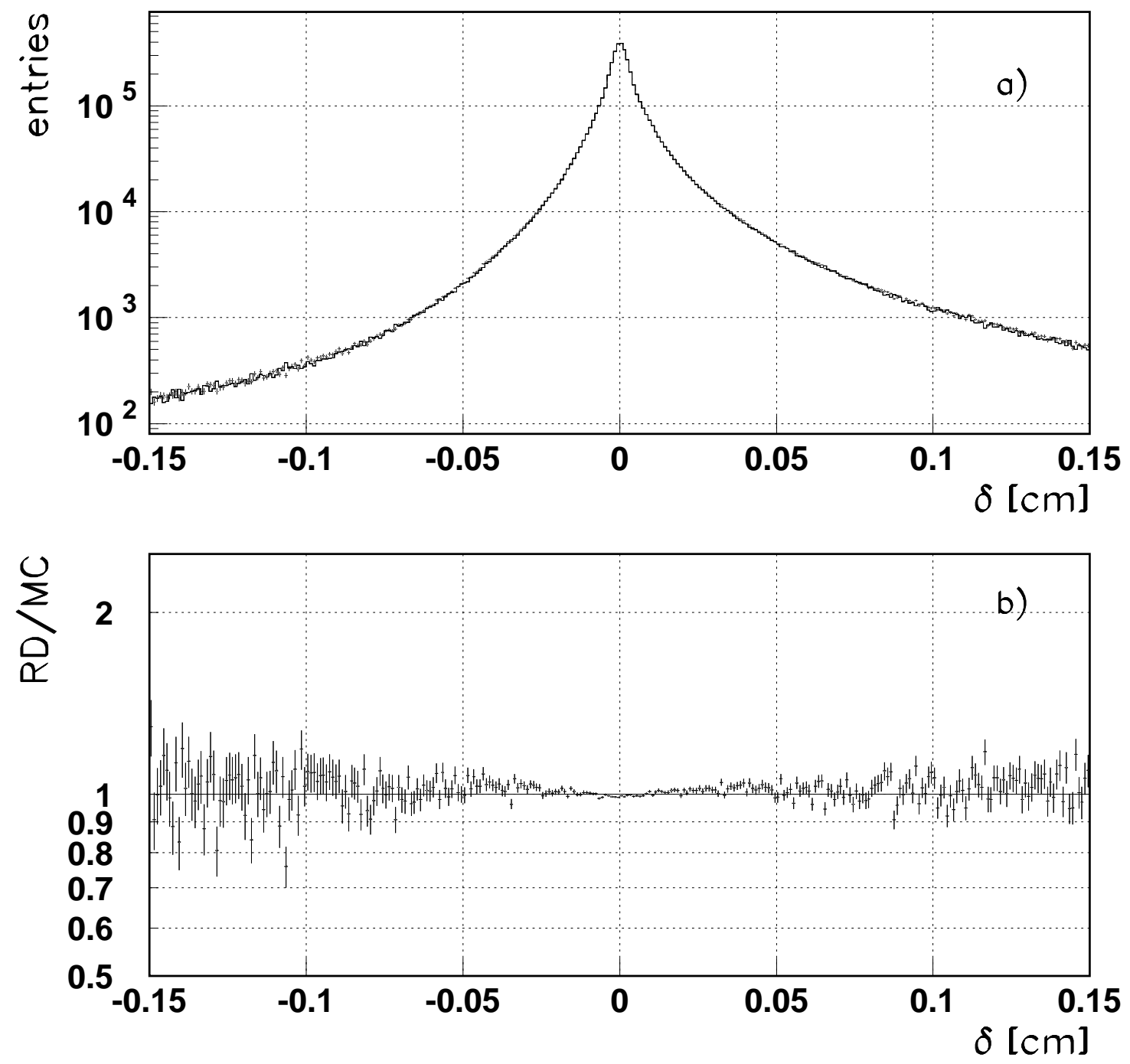

Figure 10: 

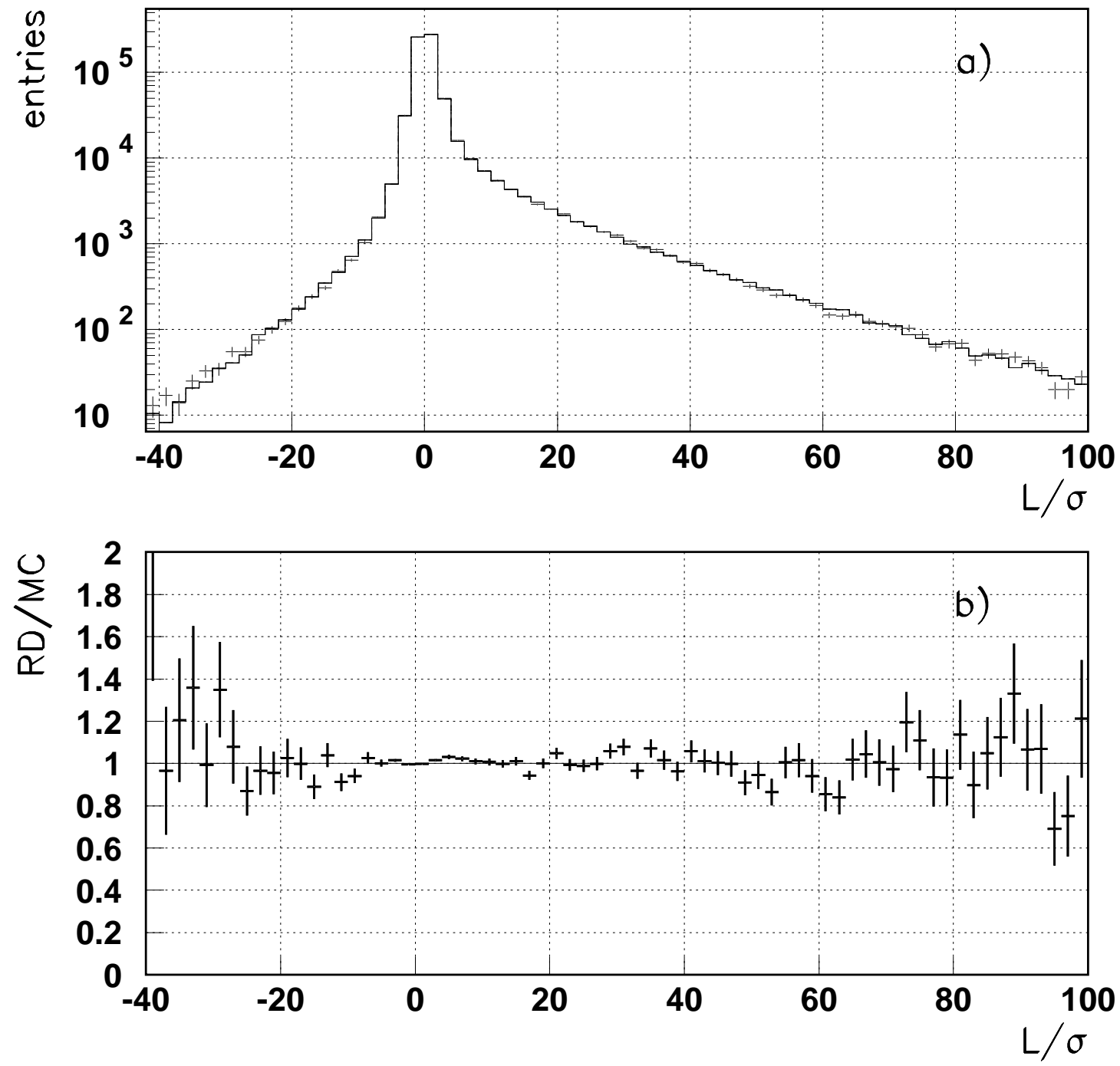

Figure 11: 